\title{
General health education as the main approach to leprosy control, Dichpalli, India
}

\author{
L M HOGERZEIL \& P KESAVA REDDY \\ Victoria Hospital Dichpalli 503 175, Nizamabad District, India
}

Received 8 January 1982

Summary Leprosy control in an integrated Community Health Project (CHP) and in a conventional Survey, Education, Treatment (SET) programme are compared over a period of 5 years.

In the CHP priority was given to intensive, continuous health education on various subjects, including leprosy, by mainly illiterate Village Health Workers (VHW), 1 per 1,000 population.

In the SET programme the emphasis was on house-to-house survey for leprosy patients only by well educated paramedical workers, 1 per 20,000 population.

Case finding in the SET was better than in the CHP. However, while the number of new patients in the SET remained fairly constant over the years, it more than doubled in the CHP. Case holding in the SET gradually increased to $64 \%$ of the registered patients receiving regular treatment, while in the CHP the corresponding figure rose to $90 \%$.

The integrated approach with the emphasis on health education seems to lead to a better quality contact between the VHW and the leprosy patient. Supervised combined therapy of all patients, tuberculoid and lepromatous, in accordance with the latest principles is now a distinct possibility.

\section{Introduction}

The National Leprosy Control Programme SET is a good programme. It has been shown that in competent hands it can control, if not eradicate leprosy. There are, however, some weaknesses in the scheme and as a result in many places leprosy has not been controlled.

The causes of failure of a leprosy control programme are, of course, well known. There is in the first place the general public's ignorance and superstition, causing the patient to evade investigation of his disease at an early 
stage. As a result case finding is delayed which may lead to disability of the patient and continued spread of the infection among the population. Then there is our inability to keep the patient on regular treatment for many years or even a life time. This inadequate case holding may also lead to disability, spread of the infection and, worst of all, to sulphone resistance. In both cases, detecting patients in an early stage of their disease and keeping them on prolonged regular treatment, the crucial factor for success in controlling leprosy is the availability of devoted staff.

In this paper we shall outline some ways in which we have been trying to tackle the problems mentioned above in our leprosy control programme at Dichpalli.

\section{Integration}

In 1963 we were requested by the State Government to take up leprosy control in an area of 1 lakh population. During the next 10 years the work was done in strict accordance: with the guide lines of the National Leprosy Control Programme. Unfortunately, the results were not very encouraging and for this we mainly blamed the general public's prejudiced attitude.

So the first thing we did was to try to reduce the stigma of leprosy by setting up an integrated CHP. This provides general health services to a population of 40,000. For the sake of comparison the National Leprosy Control programme was continued among the remaining 60,000 population.

\section{Health Education}

Very soon we discovered that in the integrated CHP health education was the first priority. It motivates people to accept immunizations, family welfare, tuberculosis- and leprosy-control programmes and so on. We also quickly found out that health education is a continuous process: once begun, it should never be stopped. To have its full impact it should be done in a leisurely fashion, on a person-to-person basis, and therefore, it takes far more time than the curative part of the project. But we believe that this is time well spent as in the end chronic diseases like leprosy and tuberculosis can only be treated satisfactorily if the patients fully understand the nature of their disease.

\section{The Village Health Worker}

Who are the most suitable people to impart health education to the community? In our opinion paramedical workers are overqualified for this kind of work. 
They do not use the same vocabulary as the village people, most of whom are illiterate, and therefore no real communication is possible. Moreover, they are considered outsiders and usually do not enjoy the full confidence of the community. We therefore prefer as health educators local women, selected by the Village Health Committee which consists of non-formal leaders identified through a 'hidden leaders survey'. Nearly all our VHWs are illiterate. They are married, have children and are usually employed. Their work as health educator has to be part time, 2 hours in the evening, and if possible their salaries are met by the Village Health Committee.

The advantages of these local VHWs are obvious: by selecting and occasionally by paying them participation of the community is ensured. There are no problems of accommodation, transport, transfer or promotion. And most important of all, the VHWs turn out to be just the devoted type of workers which are so crucial for the success of any health programme, because they are well motivated being part of the community and sharing their problems. We aim at 1 VHW per thousand population and have at present 30 VHWs in our project. They have been trained by us and continue their training by spending 1 day a week in the hospital. Apart from consolidating their knowledge and learning new health subjects there is an opportunity of exchanging experiences, sharing a meal and thus building up an 'esprit de corps'.

The VHWs main occupation is house visiting, 4-6 families a day. During these visits she gives health education on the subjects which she has been taught during the previous week. There are 16 different subjects, e.g. care of children under 5, family welfare, water-borne diseases, hygiene, eye diseases, skin diseases and leprosy. The VHW spots new patients during her house visits and refers them to the weekly village clinic conducted by a hospital nurse who gives immunizations and simple treatment. Problem patients are referred to the hospital. The VHW has no curative task, but she does remind patients suffering from chronic disease like leprosy, tuberculosis, diabetes, hypertension and epilepsy when they are due for their next check-up and treatment.

\section{Results}

CASE FINDING OVER A PERIOD OF 5 YEARS

New leprosy patients annually registered

in the CHP area, population 40,000: $12-20-24-31-29$

in the SET* area, population 60,000: 69-30-66-33-62

In the CHP area all new patients presented themselves spontaneously for examination and treatment. In the SET area most new patients were found during house-to-house survey.

* Survey, Education, Treatment i.e. the National Leprosy Control Programme. 
Note: The fluctuation in the SET figures may be explained by transport problems which were more serious in the SET area than in the CHP area.

\section{CASE HOLDING OVER A PERIOD OF 5 YEARS}

Leprosy patients receiving regular treatment ( $75 \%$ or more) in the CHP area 33-22-53-86-90\% of all registered patients in the SET area $28-50-78-51-64 \%$ of all registered patients

Note: The total number of leprosy patients registered on 1-1-1980 was 186 in the CHP area 799 in the SET area. On 1-1-1982 (2 years after completing this study) these numbers were 166 for the CHP area and 384 for the SET area.

\section{Discussion}

Five years is a short period for comparison between 2 leprosy control programmes, especially as the number of patients is rather small. Thus we should be cautious in drawing our conclusions. However, certain trends seem to be emerging.

\section{CASE FINDING}

The SET programme has been better than in the CHP, as could be expected from a programme concentrating exclusively on the detection of leprosy patients. At the same time it should be noticed that in the SET programme case finding remained fairly constant, while in the CHP there was an increase of nearly $150 \%$. If this trend in the CHP should continue, case detection by health education only might in the near future overtake case detection by mass survey in the SET programme. However, it should be pointed out that better case finding is only of value in so far as it is matched by better case holding.

\section{CASE HOLDING}

This seems to have fared much better in the CHP than in the SET programme with a steady increase of regularity from 33 to $90 \%$ in the CHP as compared with an increase from 28 to $64 \%$ in the SET programme.

The time seems to have come to ask ourselves: Which of these three: Survey, Education or Treatment should be given priority?

In our opinion there is no doubt that intensive, continuous health education should have the highest priority in any leprosy control programme. Not only does it motivate the patient to present himself voluntarily to the VHW for examination but it also prompts him to continue his treatment regularly. 
Surveys seem to be of limited value as so many leprosy patients found in this way tend to default within a few months after registration.

As regards integration, there is little doubt that leprosy has to be included in general health education as it is impossible for our VHWs to teach continuously 1 subject, i.e. leprosy only. Moreover, within a short time this would single them out as the leprosy workers in the village.

Integration of leprosy surveys into general health surveys makes also good sense. Not only does it obviate the stigma of leprosy but it also leads to much more efficient use of staff, transport and funds. We firmly believe in annual surveys by VHWs together with hospital staff to assess the general health situation in the villages and to evaluate our work. In addition, every family is visited once in 2 months by the VHW who is always on the look out for new cases of leprosy.

As regards treatment the advantages of integration are not nearly so obvious. Experience in various countries has shown that integration of treatment often leads to deterioration in the care of leprosy patients. Because of the heavy workload and lack of knowledge of leprosy, these patients tend to be the first to be neglected in integrated programmes. At Dichpalli very few leprosy patients regularly attend a general village clinic. Most of them prefer confidential treatment at home from hospital staff. As the prevalence of leprosy in our area is less than 10 per 1,000 each VHW has not more than 10 leprosy patients to supervise. It is quite easy for her to check regularly on the patients' compliance with treatment and to keep in touch with those patients who have been released from control. Therefore, combined treatment for all patients, and short-term treatment of BT and TT patients has become a distinct possibility.

Finally a few words about the economics of the integrated programme. The cost of the CHP works out at approx. 5 times the cost of SET per head of population. However, the extra expenditure involved is more than offset by bringing leprosy back into the mainstream of general medicine and by the additional services provided to the community, notably under-5 clinics, antenatal care, family welfare, control of tuberculosis and simple low cost general health care.

\section{Acknowledgements}

The CHP was supported by grants from OXFAM. We are grateful to all who were involved in the CHP and SET work, in particular Bas and Joke Bueno de Mesquita and R. Surya Rao. We thank Pieter Coenen and Bernard van Drenth for the data regarding case finding and case holding. 